PAPER

\section{Characteristics of a novel nanosecond DBD microplasma reactor for flow applications}

To cite this article: A Elkholy et al 2018 Plasma Sources Sci. Technol. 27055014

View the article online for updates and enhancements.
Related content

\begin{tabular}{l} 
- Exciplex emission induced by \\
\hline nanosecond-pulsed microdischarge arrays \\
operating at high repetition rate frequency \\
Virginie Martin, Gérard Bauville, Michel \\
Fleury et al. \\
- Atmospheric pressure discharge filaments \\
\hline and microplasmas: physics, chemistry and \\
\hline diagnostics \\
Peter Bruggeman and Ronny \\
- Effect of air flow on the micro-discharge \\
\hline dynamics in an arrav of integrated coaxial \\
microhollow dielectric barrier discharges \\
Gaurav Nayak, Yanjun Du, Ronny \\
Brandenburg et al.
\end{tabular}




\title{
Characteristics of a novel nanosecond DBD microplasma reactor for flow applications
}

\author{
A Elkholy ${ }^{1,3}$ (1), S Nijdam ${ }^{2}$ (1), E van Veldhuizen ${ }^{2}, \mathbf{N}$ Dam $^{1}, \mathbf{J}$ van Oijen ${ }^{1}$, \\ U Ebert ${ }^{2}$ and L Philip H de Goey ${ }^{1}$ \\ ${ }^{1}$ Department of Mechanical Engineering, Eindhoven University of Technology, The Netherlands \\ ${ }^{2}$ Department of Applied Physics, Eindhoven University of Technology, The Netherlands \\ ${ }^{3}$ Department of Mechanical Engineering, Helwan University, Egypt \\ E-mail: A.elkholy@tue.nl
}

Received 5 January 2018, revised 29 March 2018

Accepted for publication 19 April 2018

Published 21 May 2018

\begin{abstract}
We present a novel microplasma flow reactor using a dielectric barrier discharge (DBD) driven by repetitive nanosecond high-voltage pulses. Our DBD-based geometry can generate a nonthermal plasma discharge at atmospheric pressure and below in a regular pattern of microchannels. This reactor can work continuously up to about $100 \mathrm{~min}$ in air, depending on the pulse repetition rate and operating pressure. We here present the geometry and main characteristics of the reactor. Pulse energies of 1.46 and $1.3 \mu \mathrm{J}$ per channel at atmospheric pressure and 50 mbar, respectively, have been determined by time-resolved measurements of current and voltage. Time-resolved optical emission spectroscopy measurements have been performed to calculate the relative species concentrations and temperatures (vibrational and rotational) of the discharge. The effects of the operating pressure and flow velocity on the discharge intensity have been investigated. In addition, the effective reduced electric field strength $(E / N)_{\text {eff }}$ has been obtained from the intensity ratio of vibronic emission bands of molecular nitrogen at different operating pressures and different locations. The derived $(E / N)_{\text {eff }}$ increases gradually from about 550 to $4600 \mathrm{Td}$ when decreasing the pressure from 1 bar to 100 mbar. Below 100 mbar, further pressure reduction results in a significant increase in $(E / N)_{\text {eff }}$ up to about $10000 \mathrm{Td}$ at 50 mbar.
\end{abstract}

Keywords: microplasma, DBD microplasma, atmospheric pressure plasma, plasma spectroscopy

\section{Introduction}

Plasma flow reactors can be used to chemically process a gas. They are of interest because of their high energy efficiency and high selectivity. Therefore, they have a strong potential in applications such as surface treatment [1], thin-film deposition [2], detoxification of gaseous pollution [3, 4], plasma medicine [5] and ozone generation [6]. Many different designs have been tried to generate a stable non-thermal plasma discharge in a flow reactor at pressures up to one atmosphere. Some of these designs are plasma jets [7, 8], packed-bed plasma reactors $[9,10]$, pulsed-corona plasma reactors [11-13] and atmospheric pressure glow discharges [14].

One of the most extensively studied configurations is an array of so-called microhollow cathode discharges (MHCDs), which has the ability to produce a high-density plasma discharge in a relatively small volume (characteristic dimension of about $10^{-4} \mathrm{~m}$ ) [15-17]. Based on that, compared to other types of flow reactors, MHCD ensures a maximum interaction between the plasma discharge and the processed gas. However, two conditions should be fulfilled to maintain a discharge in MHCD devices. First, the applied voltage should exceed the breakdown voltage and be able to ignite the discharge for the given gas and pressure [18]. Second, according to the Allis-White similarity law, the product of the pressure $(p)$ and the aperture diameter of the cathode $(D)$ should fall in the typical operating range for MHCD, which is $0.1-13$ mbar $\cdot \mathrm{cm}$ depending on the gas, electrodes and geometry [19]. These conditions pose some constraints regarding scaling-up the diameter of the MHCD devices at high pressures. Based on the upper limit of the product $(p \cdot D)$, atmospheric pressure operation in air would lead to a maximum diameter $(D)$ of $100 \mu \mathrm{m}$. However, in many applications, especially for gas 


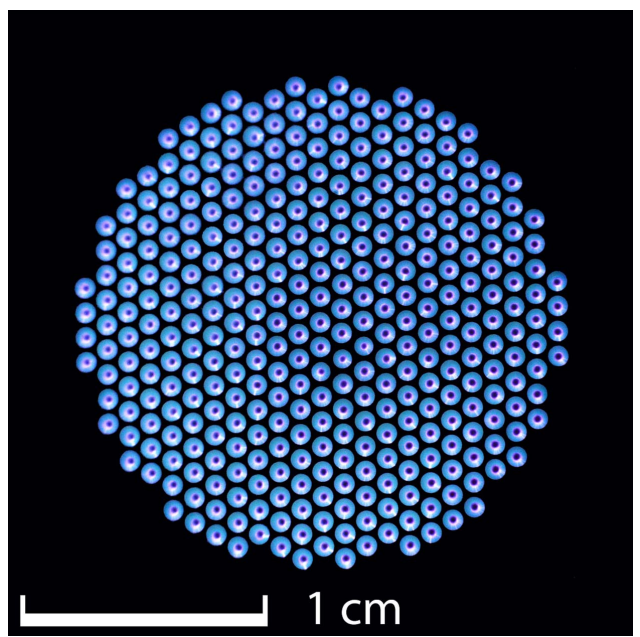

Figure 1. Image of the plasma discharge in the DBD microplasma reactor (see figure 3 for details) in air with a flow rate of $3.41 \mathrm{~min}^{-1}$, at atmospheric pressure, $5 \mathrm{kV}$ voltage pulse with a repetition frequency of $3 \mathrm{kHz}$, exposure time: $1 / 20 \mathrm{~s}$.

treatment, it is desirable to increase the diameter to reduce the pressure drop for the same flow condition.

One of the most successful ways to increase the hole diameter of the reactor is by using a dielectric barrier discharge (DBD) [20, 21] driven by nanosecond high-voltage pulses. The surface discharge deposited by the (pulsed) discharge counteracts the applied field, thereby self-limiting the discharge. Together with the nanosecond pulses, this helps to generate a uniform discharge distribution and reduces the chance of glow-to-arc transition. In addition, using nanosecond repetitive high-voltage pulses provides highly energetic electrons and more chemically excited species compared to AC power sources due to the rapid ionization process, with a power consumption that is about one order of magnitude lower [22, 23]. The increase of the electron energy comes without a considerable increase of the gas temperature, thereby reducing undesired thermal effects of the plasma discharge on the treated gas.

In this paper, we introduce a new geometry, which utilizes the DBD method to sustain a non-thermal plasma discharge in an array of $400 \mu \mathrm{m}$ diameter channels perforating a $1.5 \mathrm{~mm}$ thick dielectric slab with embedded electrodes. The discharge is powered by nanosecond high-voltage pulses and is operated at pressures up to one atmosphere, which corresponds to a pressure $(p)$ times diameter $(D)$ of $40 \mathrm{mbar} \cdot \mathrm{cm}$. The main development in this study is that the high-voltage electrode is fully embedded within the dielectric substrate. This allows us to generate a non-thermal plasma discharge at atmospheric pressure in channels larger than the maximum possible diameter for MHCDs at the same pressure.

The geometry consists of 363 channels of $400 \mu \mathrm{m}$ in diameter placed in parallel. Each channel has two sets of electrodes, stacked to work (optionally) in series to increase the discharge volume in order to increase the efficiency of the gas-discharge interaction. Figure 1 shows a top-view luminosity image of a plasma discharge in this DBD microplasma reactor, with $3.41 \mathrm{~min}^{-1}$ air flow upwards, out of the plane of

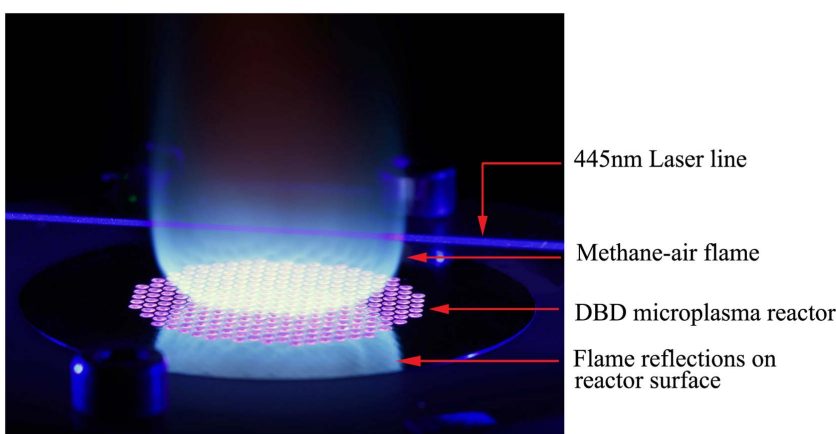

Figure 2. Image of a methane-air flame stabilized on the DBD microplasma reactor at atmospheric pressure. Laser is used for flame diagnostics and is not discussed in this paper.

the paper, at atmospheric pressure, $5 \mathrm{kV}$ voltage pulse with a repetition rate of $3 \mathrm{kHz}$, recorded by a digital DSLR camera (Nikon D5100).

The non-thermal nature and the high electron energy of the produced discharge make this geometry very promising for applications such as plasma-assisted combustion, chemical treatment, light sources, plasma medicine, pollutant control and many others.

In this paper, we present the geometry, material and fabrication process of the DBD microplasma reactor as well as the characteristics of the nanosecond pulsed plasma produced by it. Time-resolved optical spectroscopy is used to investigate the discharge emission and its temperatures. ICCD images are analyzed to identify the discharge structures at different operating pressures. The relation between the effective reduced electric field strength $(E / N)_{\text {eff }}$ and operating pressure and the effect of flow velocity on discharge emission are also presented. Finally, the reactor lifetime is determined for different operating pressures and pulse repetition rates.

An example of the use of our reactor is given in figure 2 where it stabilizes a methane-air flame in order for us to study the effect of the plasma discharge on combustion characteristics. The preliminary results of this study show a significant increase, reaching up to $100 \%$ in some cases, in the laminar burning velocity as a result of the plasma discharge. Results of this combustion study will be published within a different framework.

\section{Experimental setup}

\subsection{Reactor geometry, materials and fabrication}

As shown in figure 3, the new plasma device is a $1.5 \mathrm{~mm}$ thick dielectric slab perforated by a hexagonal array of 363 channels. The channels have a diameter of $0.4 \mathrm{~mm}$ and a pitch of $1.0 \mathrm{~mm}$. The outer diameter of the reactor is $70 \mathrm{~mm}$, while the effective diameter of the flow area is $20 \mathrm{~mm}$. The reactor geometry includes four layers of copper electrodes (A, B, C and $\mathrm{D}$ ) in figure $3 \mathrm{~B}$. The high-voltage electrodes ( $\mathrm{B}$ and $\mathrm{C}$ ) are embedded into the dielectric material. The grounded electrodes (A and D) are located on the top and bottom 


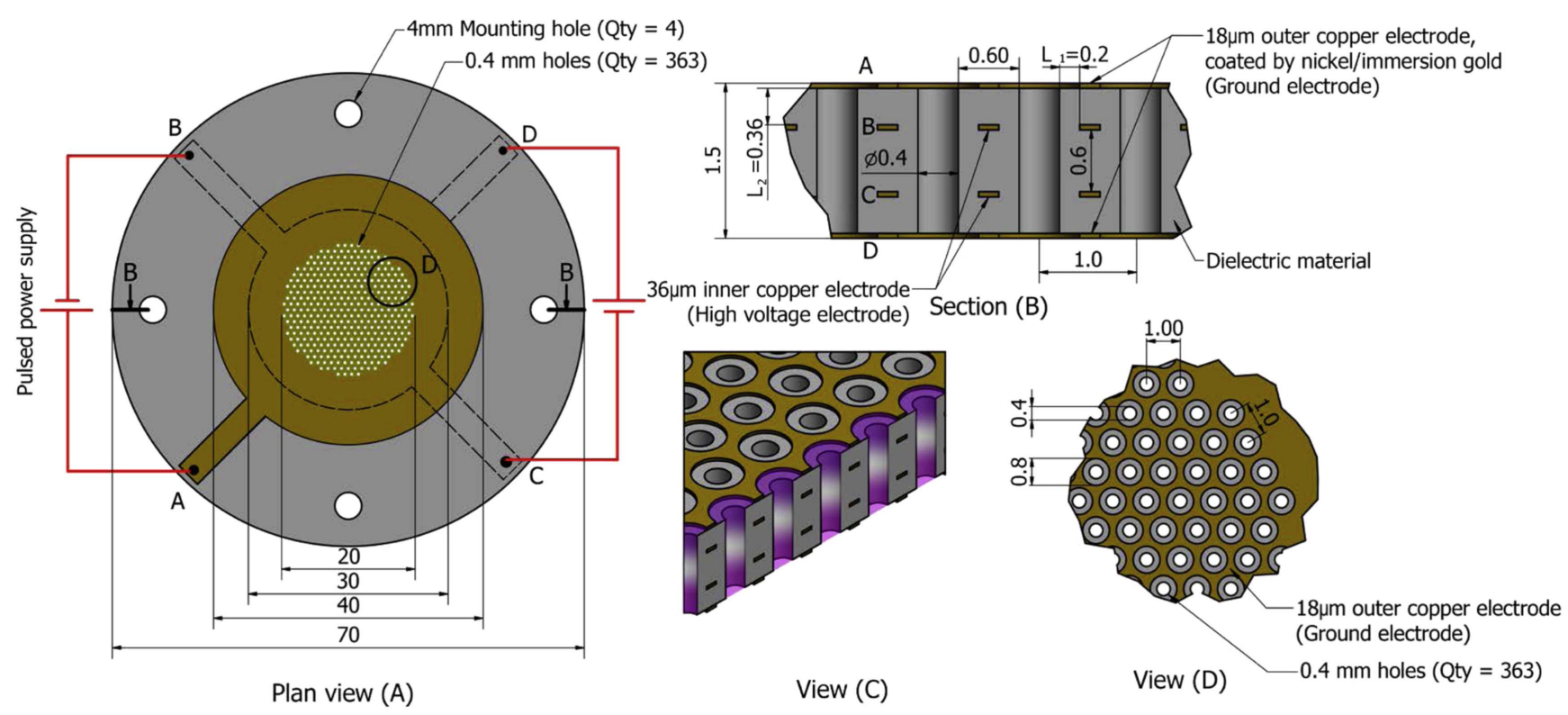

Figure 3. Schematic of the reactor configuration. All dimensions are in $\mathrm{mm}$.

surfaces of the reactor. The outer and the inner copper layers have a diameter of 40 and $30 \mathrm{~mm}$, respectively. All electrodes are perforated in the same hexagonal pattern as the dielectric, but the holes are twice as large as those in the dielectric. The device can work in two modes: single-layer discharge mode and double-layer discharge mode. In the single-layer discharge mode, only electrodes $\mathrm{A}$ and $\mathrm{B}$ are activated (see figure 5 for the equivalent circuit diagram), while electrodes $C$ and $\mathrm{D}$ are kept floating. All the work presented in this study has been done in the single-layer discharge mode.

The cathode and anode are separated by a dielectric layer with a thickness of $0.36 \mathrm{~mm}$. The outer (grounded) and inner (high-voltage) electrodes are made of copper with thicknesses of 18 and $38 \mu \mathrm{m}$, respectively. The separation distance between the two embedded electrodes is $0.6 \mathrm{~mm}$, while the total thickness of the reactor is $1.5 \mathrm{~mm}$. The width and thickness of the embedded electrodes have been calculated to safely withstand the peak current of the discharge pulses, which is $205 \mathrm{~mA}$ per channel for a duration of $10 \mathrm{~ns}$, as shown in figure 7 (a).

We came to this design by performing a geometrical parameter study to (i) determine the minimum dielectric thickness in order to maximize the discharge strength and (ii) guide most of the discharge to the inside of the holes instead of the top or bottom reactor surfaces, to get an efficient interaction with the flowing gas. From this study we found that the dielectric thickness between the anode and the wall of the hole, $\left(L_{1}\right.$ in view (B) of figure 3$)$, should be less than the axial dielectric thickness between the anode and cathode $\left(L_{2}\right)$.

The dielectric material of the reactor is a composite of woven electrical grade fiberglass and epoxy resin. It has a dielectric constant $D_{r}$ of $4.17 \pm 0.05$ (at $1 \mathrm{GHz} / 23{ }^{\circ} \mathrm{C}$ ), a thermal conductivity of $0.4 \mathrm{~W} \mathrm{~m} \cdot \mathrm{K}^{-1}$, a dielectric breakdown strength of $32 \mathrm{kV} \mathrm{mm}^{-1}$, a thermal expansion coefficient of $14 \mathrm{ppm} /{ }^{\circ} \mathrm{C}$ and a glass transition temperature of $180{ }^{\circ} \mathrm{C}$. This material has a stable performance over a wide range of frequencies and temperatures, as well as good machinability.

In order to reduce the chance of micro-filamentary discharges on the reactor surface, which would reduce the reactor lifetime as a result of excessive heat, the outer copper electrodes have been coated with a nickel/immersion gold layer, which gives a surface roughness of $0.5 \mu \mathrm{m}$. In some applications, which are accompanied by thermal radiation, such as plasma-assisted combustion or chemical vapor deposition, the copper layers also act as protective layers to conduct away excessive heat from the process. This prevents overheating of the dielectric material, which can lead to dielectric failure.

The plasma reactor is manufactured by a multi-step UV lithography process. First, the desired copper patterns are precisely defined on two dielectric slabs with copper layers pre-bonded onto each side. These correspond to the outer and inner electrode pairs and will be stacked on top of each other later in the process. Then, UV light is used to harden a photoresist layer on the desired copper tracks, after which an alkaline solution is sprayed over the panel to etch away the undesired copper. Next, the two panels are stacked together bonded by another epoxy resin layer and thermally pressed to guarantee a permanent bond without any air gap. Then, the copper tracks on the upper and lower layers are coated with a nickel/immersion gold layer. Finally, the channels are drilled with a laser drilling machine to ensure clean hole walls and a uniform pattern.

\subsection{Flow setup}

A schematic representation of the experimental setup is shown in figure 4 . The setup consists of three main parts; the plenum chamber at the bottom, a water jacket in the middle and the microplasma reactor on top. The plenum chamber and the water jacket are made of steel and brass, respectively. The 


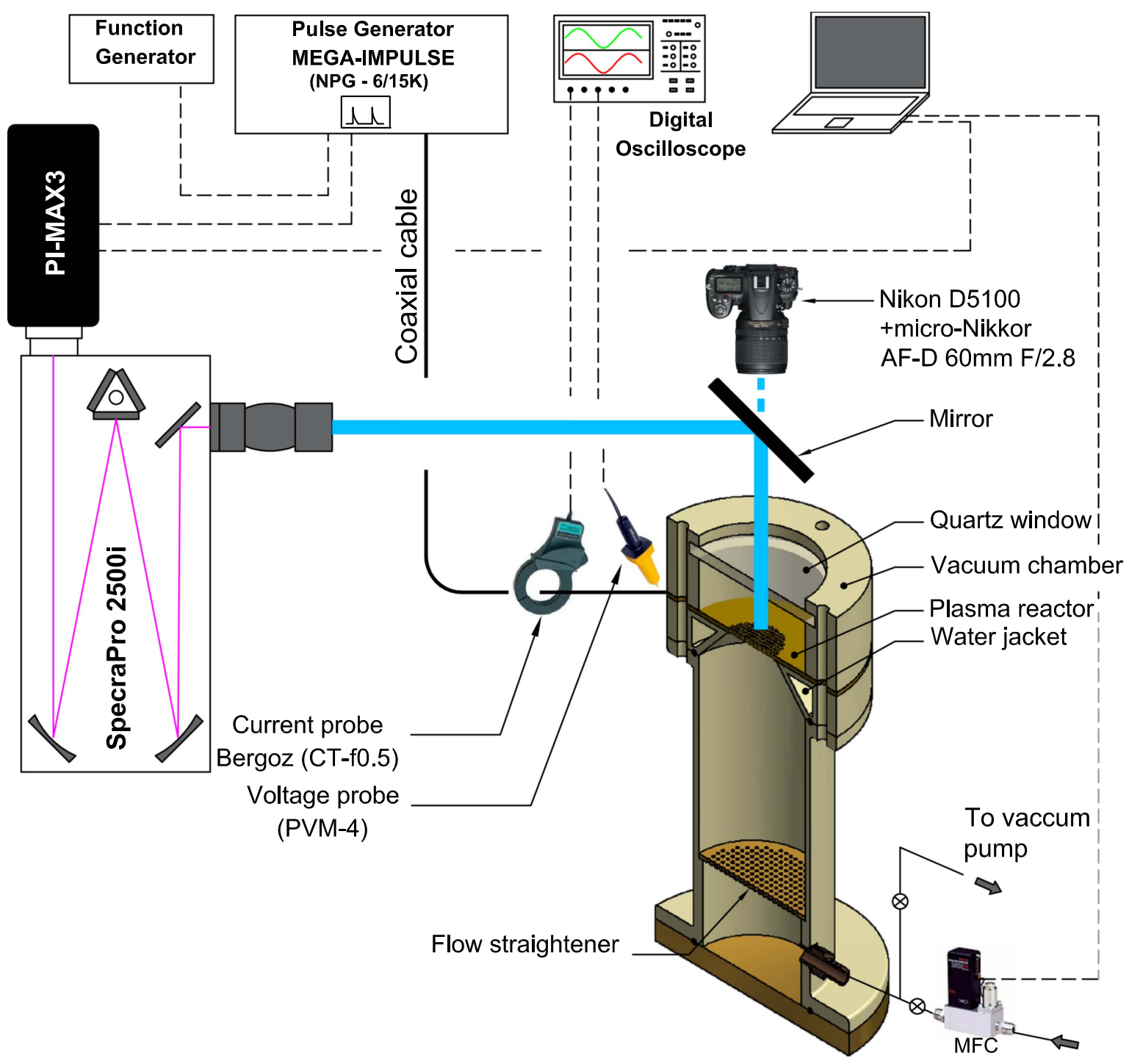

Figure 4. Schematic of the experimental setup.

purpose of these parts is to create a uniform air flow towards the plasma reactor. The gas inlet port is located at the bottom of the plenum chamber. A perforated plate at $20 \mathrm{~mm}$ from the bottom works as a flow straightener. The holes in the perforated plate $(1.0 \mathrm{~mm}$ in diameter and $2.0 \mathrm{~mm}$ in pitch) cover the entire area of the plate. In case of reduced pressure, a vacuum chamber of $20 \mathrm{~mm}$ height and $60 \mathrm{~mm}$ in diameter is mounted on top of the plasma reactor. The top flange of the chamber includes a fused quartz window with a thickness of $4 \mathrm{~mm}$, through which the discharge emission can be viewed. The reactor temperature is controlled by means of the water jacket underneath the reactor. The water inlet and outlet are connected to a heater and thermostat to control the water temperature. This water circuit can heat up and control the upper surface of the reactor up to $80^{\circ} \mathrm{C}$.

The air flow is controlled by a mass flow controller (MFC; Bronkhorst model F-202CV). A cylindrical buffer vessel is placed before the MFC to damp pressure fluctuations. In addition, an air filter is installed just before the MFC to avoid contamination. To ensure purity, synthetic air of grade 4.0 has been used. A vacuum pump model Busch R5 KB 0010, with a minimum pressure of 20 mbar and flow of $10 \mathrm{~m}^{3} \mathrm{~h}^{-1}$, has been used to evacuate the vacuum chamber to the desired pressure.

\subsection{Electrical characteristics}

Figure 5 shows the equivalent electrical circuit diagram as used with single-layer operation (red box) and double-layer operation (blue box) for the DBD microplasma reactor. In the case of double-layer operation, the total voltage $V_{(\mathrm{t})}$ is the same for the two layers, while the current is divided evenly between the two layers. When the voltage exceeds the breakdown voltage, indicated as an ignition switch in the figure, the current $I_{D}$ will pass through the discharge gap to initiate the plasma discharge.

The high-voltage pulses are produced by a Mega-Impulse semiconductor-based pulse generator, model NPG-6/15 k. Positive polarity pulses of $4-7 \mathrm{kV}$ in amplitude, $25 \mathrm{~ns}$ in duration and $10 \mathrm{~ns}$ rise time are created at a pulse repetition frequency in the range of $0-10 \mathrm{kHz}$. The pulse generator is 


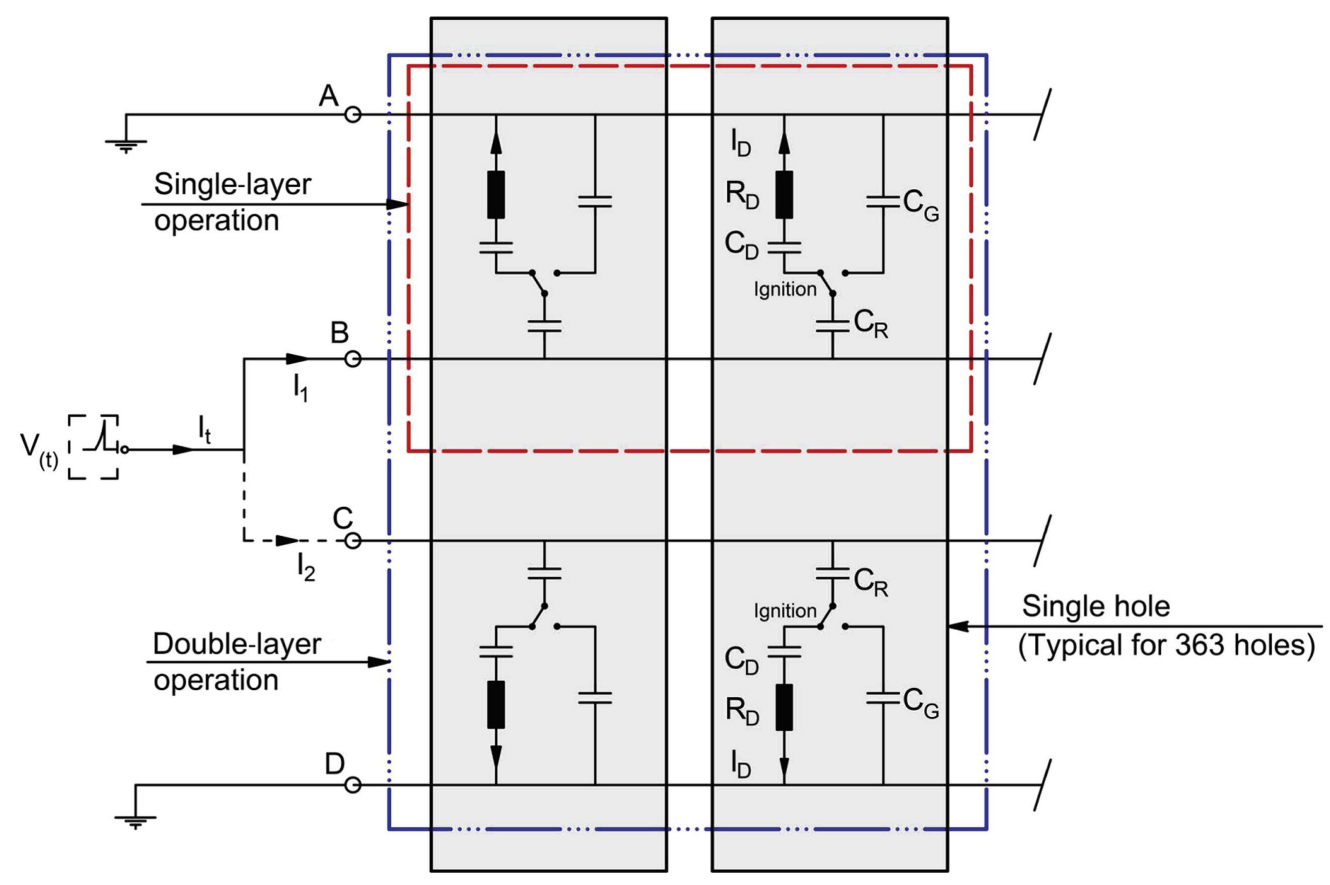

Figure 5. Equivalent electrical circuit diagram for single-layer operation (red box) and double-layer operation (blue box). For connections A, $\mathrm{B}, \mathrm{C}$ and D, refer to figure $3 . C_{\mathrm{R}}$ is the reactor capacitance, $C_{G}$ is the air gap capacitance, $R_{\mathrm{D}}$ and $C_{\mathrm{D}}$ are the resistance and capacitance of the discharge, respectively. For illustration purposes only, two of the 363 holes are drawn.

externally triggered by a FeelTech frequency generator model FY2102S. Voltage and current are simultaneously monitored by a Lecroy waverunner 44MXi-A oscilloscope with an analog bandwidth of $400 \mathrm{MHz}$ and sampling rate of $5 \mathrm{GS} \mathrm{s}^{-1}$.

The voltage is recorded by a North Star high-voltage probe model PVM- 4 with a sensitivity of $1 \mathrm{~V} / 1 \mathrm{kV}$, a bandwidth of $120 \mathrm{MHz}$ and $2 \mathrm{~ns}$ rise time. The current through the electrodes is measured by a Bergoz current transformer model CT-F0.5 with a $1.75 \mathrm{~ns}$ rise time and a sensitivity of $0.5 \mathrm{~V} / \mathrm{A}$ voltage-to-current conversion located on the ground cable.

For precise calculation of the pulse energy, it is important to make sure that the voltage and current signals are synchronized. This was achieved by matching the calculated capacitive current from the voltage signal and the measured current for a pure capacitive case. To ensure a pure capacitive current, the applied voltage across the electrodes is set to the minimum possible value of the power supply, which is $4 \mathrm{kV}$ with a pulse repetition rate of $10 \mathrm{~Hz}$. Also, the capacitance between the electrodes has been increased by filling the channels and covering the surfaces of the reactor with silicon material, preventing a discharge from developing. The waveshape of the expected capacitive current $\left(I_{\text {cap }}\right)$ was then calculated by taking the time-derivative of the voltage signal $(V)$ multiplied by the modified capacitance of the device $\left(C_{\mathrm{mod}}\right)$ :

$$
I_{\text {cap }}=C_{\text {mod }} \frac{\mathrm{d} V}{\mathrm{~d} t}
$$

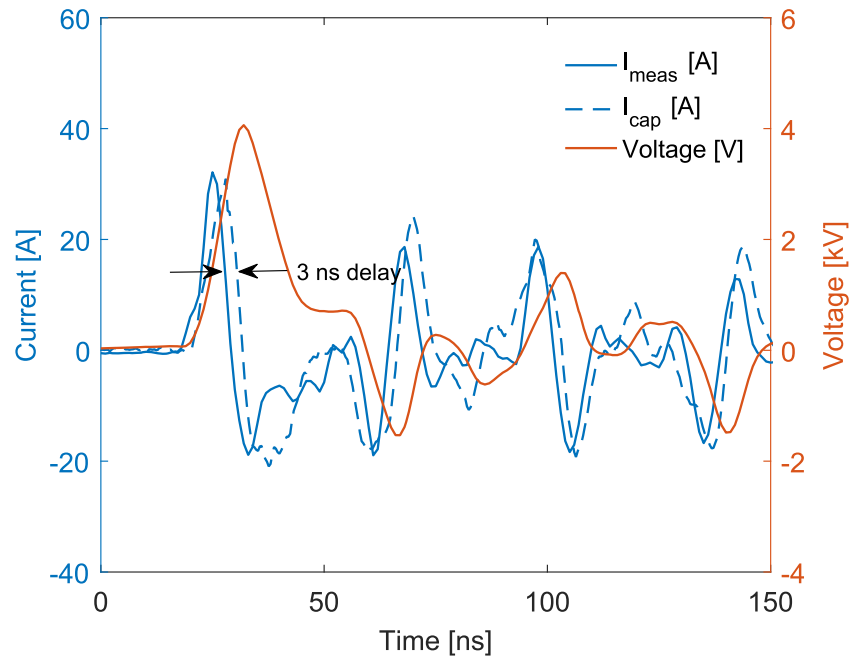

Figure 6. Measured voltage, measured current and the calculated capacitive current for the modified reactor at atmospheric pressure, voltage of $4 \mathrm{kV}$ and a pulse repetition rate of $10 \mathrm{~Hz}$.

Figure 6 shows the measured voltage and current waveforms and the calculated capacitive current, for the modified reactor. A time delay between the measured current and the calculated capacitive current of 3 ns has been found. This time delay has been added to all the measured current signals to adjust for the phase-shift.

The reciprocal of the effective capacitance $C_{\text {total }}$ is the sum of the reciprocal of two components, the reactor capacitance $C_{\mathrm{R}}$ and the cable capacitance $C_{\text {cable. }}$. Both the reactor and cable capacitances have been measured by a Fluke RCL meter model PM6303, resulting in $C_{\mathrm{R}}=94 \quad p F$ and 
Table 1. Blaze wavelength and equivalent FWHM of the slit function for a $20 \mu \mathrm{m}$ wide slit of the gratings used in this experiment.

\begin{tabular}{lccc}
\hline & \multicolumn{3}{c}{ Grating (grooves $/ \mathrm{mm}$ ) } \\
\cline { 2 - 4 } & 300 & 1800 & 3600 \\
\hline Blaze wavelength (nm) & 300 & 200 & 300 \\
FWHM (nm) & 0.58 & 0.084 & 0.037 \\
\hline
\end{tabular}

$C_{\text {cable }}=200 p F$. So, the equivalent total capacitance is $C_{\text {total }}=64 p F$.

\subsection{Optical emission spectroscopy setup}

Optical emission spectroscopy has been performed with a SpectraPro 2500i spectrometer with a focal distance of $0.5 \mathrm{~m}$, fitted with three gratings of 300,1800 and 3600 grooves $/ \mathrm{mm}$. Table 1 shows the blaze wavelengths and the equivalent full widths at half-maximum (FWHM) of the slit functions (approximately a Gaussian shape) for a $20 \mu \mathrm{m}$ wide slit measured at $355 \mathrm{~nm}$ for these gratings. The emission spectra from the plasma discharge have been recorded using a $1024 \times 1024$ pixel ICCD camera (Princeton Instruments PIMax 3) on the exit port of the spectrograph. The spectral line wavelength of the entire optical system is calibrated using the $253.65,435.83$ and $579.07 \mathrm{~nm}$ lines of a low-pressure pencil-type mercury lamp [24], model ORIEL 6035. A UVNikkor lens with $105 \mathrm{~mm}$ focal length and $\mathrm{f} / 4.5$ is attached to the spectrometer entrance slit to collect the emitted light from the plasma discharge.

Synchronization between the voltage pulse signal and the ICCD camera gate was achieved by a triggering signal from the high-voltage pulse generator, which was adjusted by the internal delay generator of the ICCD camera. A $200 \mathrm{~ns}$ time delay has been observed between the rising edge of the highvoltage pulse and the onset of the plasma discharge. This time delay comes from three sources: (1) the high-voltage pulse generator delay, (2) signal delay due to $3 \mathrm{~m}$ cable length and (3) the charging of the reactor capacitance. The digital images have been recorded using a commercial 16.2 mega-pixel digital camera Nikon D5100 fitted with a micro-Nikkor lens with a $60 \mathrm{~mm}$ focal length and $\mathrm{f} / 2.8$. The high-speed images have been recoded using a FASTCAM Mini UX100 camera fitted with an Invisible Vision intensifier model UVi 1850-05 and a $60 \mathrm{~mm}$ Nikkor micro lens. The synchronization between the image intensifier and the high-voltage pulses was achieved by a BNC-575 pulse-delay generator.

\section{Results and discussion}

\subsection{Pulse characteristics and energy calculation}

Figure 7(a) shows the current and voltage waveforms, after synchronization, of a single pulse for a single-layer operation in stagnant air at atmospheric pressure (solid lines) and at 50 mbar (dashed lines). The voltage signal shows a positive polarity incident pulse with a $5 \mathrm{kV}$ peak followed by a negative polarity reflected pulse with a $1.75 \mathrm{kV}$ peak. The rise time of the incident pulse is about $10 \mathrm{~ns}$. The current signal shows the same behavior, with a positive peak of $75 \mathrm{~A}$, corresponding to $205 \mathrm{~mA}$ per channel, followed by a negative peak of $58 \mathrm{~A}$. The FWHM duration of the current signal is 7 ns and it has a rise time of $4.4 \mathrm{~ns}$.

The instantaneous pulse power $P_{\text {pulse }}$ (blue lines in figure $7(b)$ ) has been obtained by multiplying the measured voltage $V$ with the current $I$ (after adjusting the time delay). The power has been integrated over the pulse duration to get the total energy $E_{\text {pulse }}$ deposited in the plasma discharge:

$$
E_{\text {pulse }}=\int_{t_{1}}^{t_{2}} I \cdot V \mathrm{~d} t
$$

As shown in figure 7(b) (red lines), the total energy per pulse is about $0.53 \mathrm{~mJ} /$ pulse at atmospheric pressure and 0.47 $\mathrm{mJ} /$ pulse at 50 mbar. These values correspond to pulse energy of 1.46 and $1.3 \mu \mathrm{J}$ per channel, respectively. Also, it has been observed that the incident pulse is followed by a reflected pulse after about $40 \mathrm{~ns}$, followed by some ripples. This behavior has been validated by time-resolved measurement of the discharge emission, reported in section 3.3.2 below.

\subsection{Discharge structure}

Figure 8 shows the structure of the plasma discharge inside and outside of the DBD microplasma channels at different operating pressures. The figure shows luminosity images of the plasma reactor, looking directly into the channels from above (see figure 4). The pink glow is due to the plasma, the bluish glow around it is due to the dielectric (note that the holes in the electrodes have twice the diameter of the channels). Figure 9 shows short-exposure ICCD images of single pulses in one channel under similar conditions.

At atmospheric pressure, figure 8(a), the discharge is concentrated as an annulus on the inner wall of the channel and no discharge is visible in the center of the holes. This is related to the short mean free path of the electrons due to the inelastic collisions with the neutral atoms, which narrows the discharge region. Outside the holes, a micro-filamentary discharge from the hole to the cathode ring can be observed (the white reddish lines traversing the bluish regime). The singlepulse ICCD image in figure 9(a) shows similar behavior.

At a pressure of 100 mbar, figure 8(b), the mean free path of the electrons increases, resulting in a wider and more uniform annular ring inside the holes. Also, note the absence of micro-filamentary discharges in the short-exposure image (figure 9(b)). Meanwhile, the glow discharge extends beyond the confinement of the holes, giving rise to the faint pink glow over the whole field of view. By decreasing the pressure further to 50 mbar, figures 8(c) and 9(c), the plasma discharge is capable of reaching the center and uniformly fills the whole channel. 

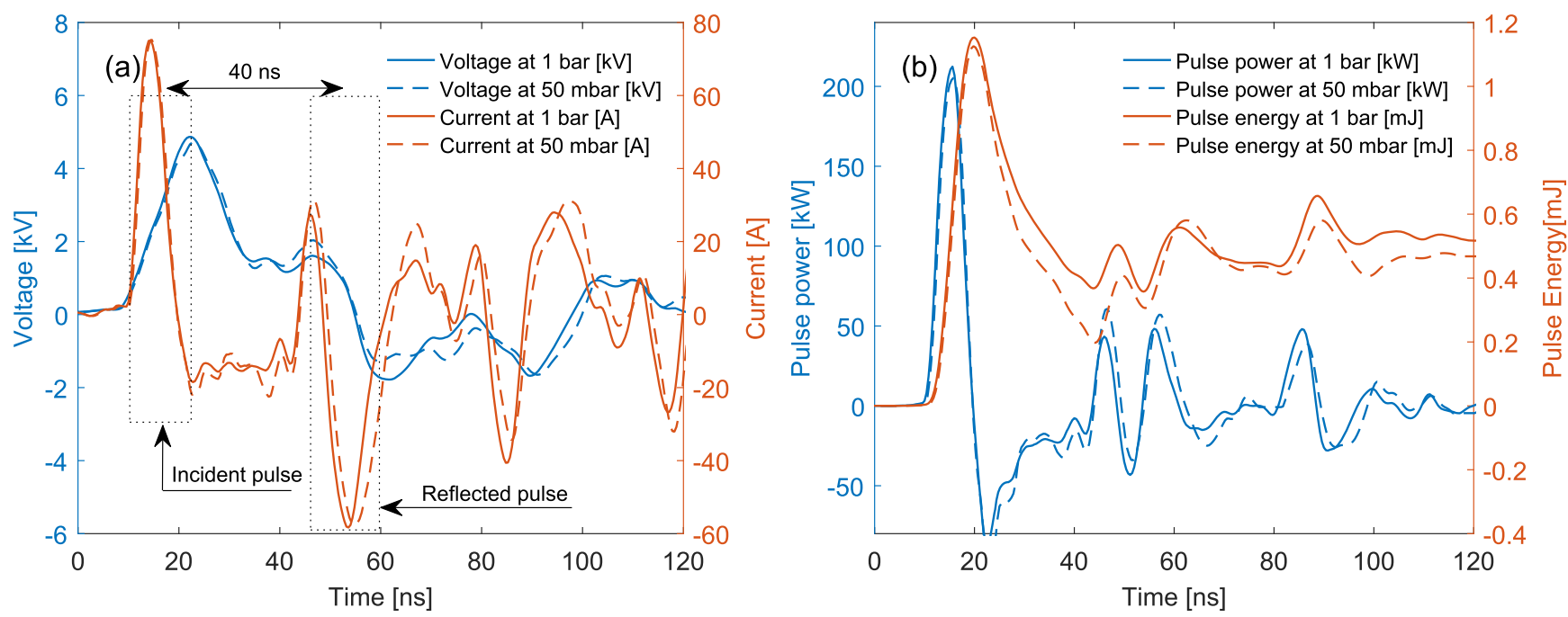

Figure 7. (a) Temporal development the applied voltage (blue lines) and resulting current (red lines) at the plasma reactor. (b) Calculated instantaneous power (blue lines) and pulse energy (red lines). All are for stagnant air at atmospheric pressure (solid lines) and 50 mbar (dashed lines).

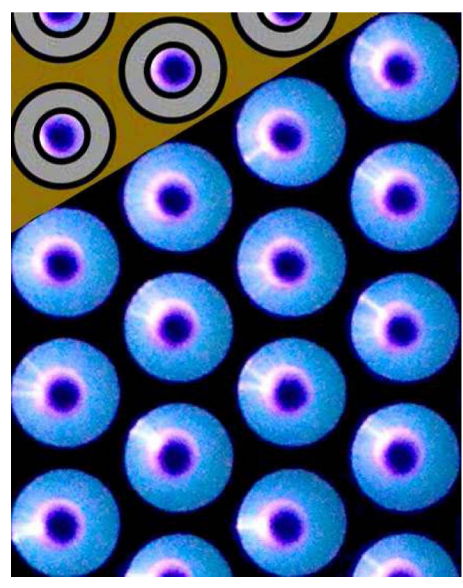

(a) $p=1$ bar

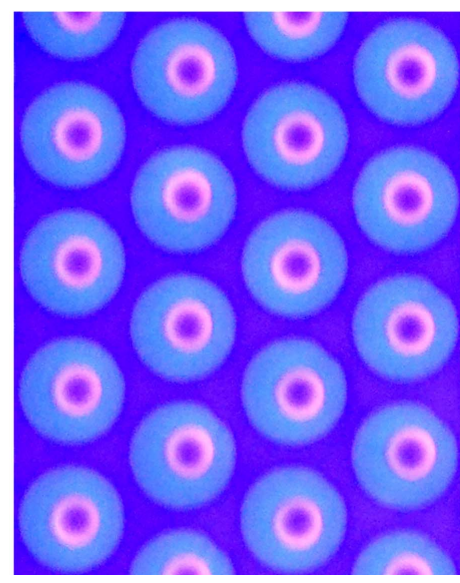

(b) $p=100$ mbar

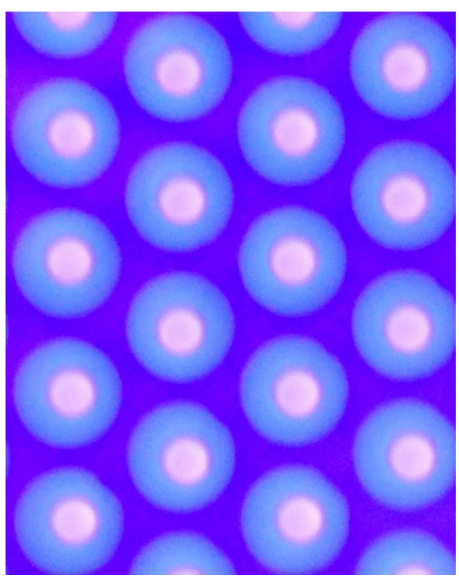

(c) $p=50$ mbar

Figure 8. Luminosity images of plasma discharge in a DBD microplasma reactor in stagnant air with a repetition frequency of $2 \mathrm{kHz}$ and $5 \mathrm{kV}$ voltage pulse at three different pressures. Exposure time $=50 \mathrm{~ms}$. The detail of the schematic drawing from figure 3D has been overlayed on (a) to give a sense of scale.

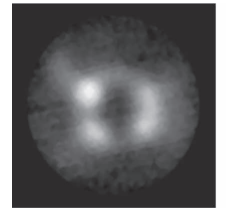

(a) $p=1$ bar

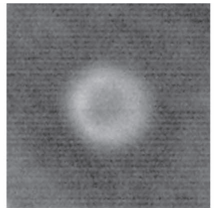

(b) $p=100 \mathrm{mbar}$

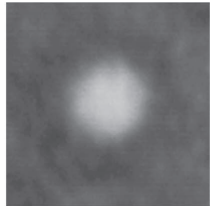

(c) $p=50$ mbar
Figure 9. ICCD images of a plasma discharge in a single channel during one pulse with an exposure time of $200 \mathrm{~ns}$ in stagnant air at $5 \mathrm{kV}$ for three different pressures.

\subsection{Temperature measurements}

Gas temperature is a very important parameter in plasma science for two reasons. First, the gas temperature has a direct effect on reactive species generation [25]. Second, there are many applications that need control of thermal behavior, such as wound treatment, chemical decomposition, combustion, etc. Nanosecond plasma discharges typically are of nonequilibrium nature resulting in different kinetic energy distributions for the different species (i.e. electrons, ions, neutrals). Apart from that, the different degrees of freedom (electronic, translation, vibration and rotation) are also not necessarily in equilibrium [26, 27]. In the following sections, we will focus on the rotational and vibrational temperature measurement of the plasma discharge in the DBD microplasma reactor, as well as the average gas temperature.

3.3.1. Rotational temperature. The rotational temperature of the plasma discharge in stagnant air has been obtained by fitting the experimentally observed spectra of the (0-2) band structure of the second positive system (SPS) of $\mathrm{N}_{2}$ $\left(C^{3} \Pi_{u} \rightarrow B^{3} \Sigma_{g}\right)$ with the Specair simulation tool [28] convoluted with the measured instrumental slit function. The spectra are integrated over $20 \mathrm{~ns}$ following the start of the voltage pulse, using a spectrograph slit width of $20 \mu \mathrm{m}$. The 

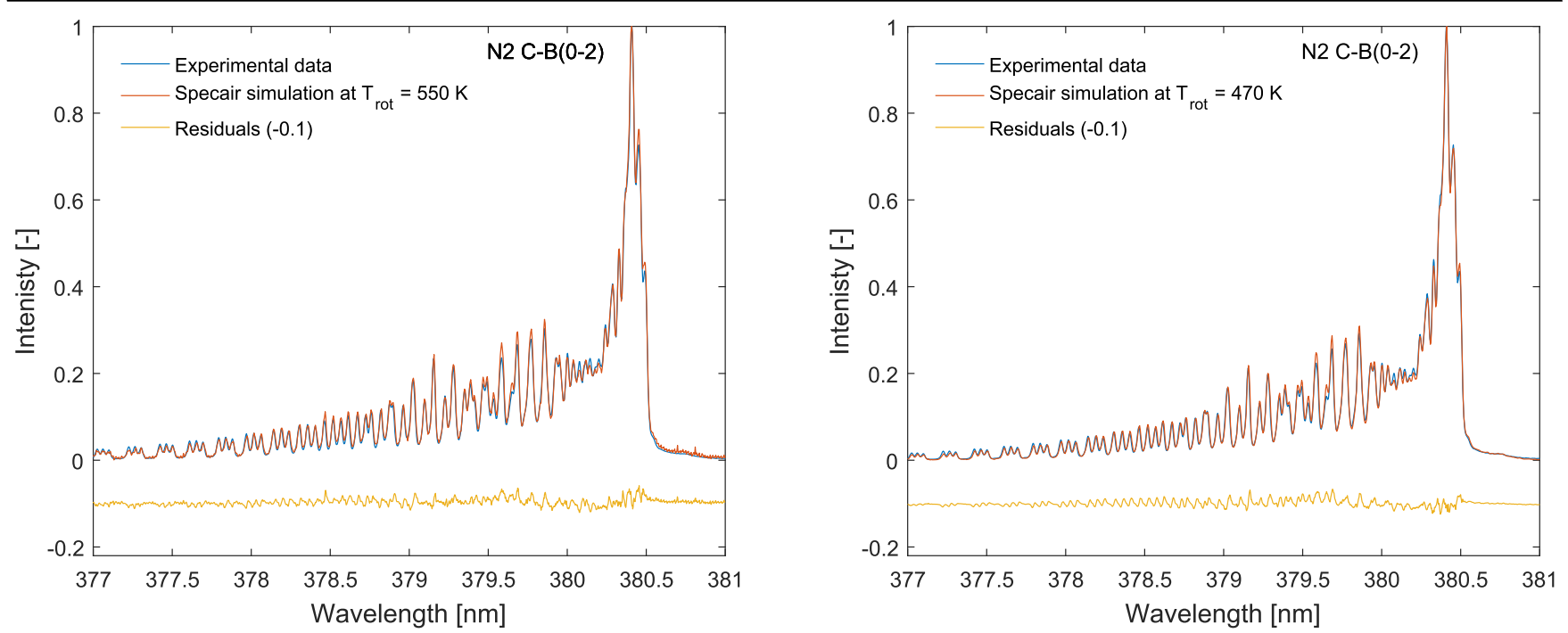

Figure 10. Experimental (blue line) and simulated (red line) normalized spectrum of stagnant air at (left) $p=1$ bar, and (right) $p=50 \mathrm{mbar}$. The simulated spectra are from Specair with $T_{\mathrm{rot}}=550 \mathrm{~K}$ and $T_{\mathrm{rot}}=470 \mathrm{~K}$, respectively. Both are for $V=5 \mathrm{kV}$ and pulse repetition rate of $3 \mathrm{kHz}$. Signals are integrated over 300,000 pulses using a 3600 grooves $/ \mathrm{mm}$ grating.

signal has been accumulated over 300,000 pulses to increase the signal-to-noise ratio. The signal was collected from an area covering four complete holes at the center of the reactor disc. The measurements are carried out for a pulse repetition frequency of $3 \mathrm{kHz}$ using the grating of 3600 grooves $/ \mathrm{mm}$.

As shown in figure 10, when comparing the rotational line structure with a simulated Boltzmann rotational distribution, a good fit was obtained at both atmospheric pressure and reduced pressure. This implies that the rotational distribution has thermalized (via heavy species collisions or electron collisions) in a short time (few nanoseconds). The rotational distribution of the excited states can be considered representative of the ground state rotational temperature, which is usually close to the gas temperature [29]. The rotational temperatures obtained from the Specair fitting procedure were $550 \mathrm{~K} \pm 30 \mathrm{~K}$ and $470 \mathrm{~K} \pm 30 \mathrm{~K}$ for pressures of $p=1 \mathrm{bar}$ and $p=50$ mbar, respectively. This reduction in the rotational temperature with pressure can be explained by the reduction in the consumed power, as shown in figure $7(\mathrm{~b})$.

3.3.2. Vibrational temperature. The vibrational temperature describes the population of the vibrational states of molecular species, if this follows a Boltzmann distribution. In this study, the vibrational temperature has been obtained by fitting the observed structure of the (0-2), (1-3) and (2-4) spectral bands of the SPS of $\mathrm{N}_{2}$ to Specair simulations. By using a grating of 1800 grooves $/ \mathrm{mm}$ and integrating the signal over 100,000 pulses.

Here, the fit is not as good as for the rotational temperature, leaving a higher uncertainty in the vibrational temperature calculations. This fitting error may be caused by errors in the intensity calibration of the spectrometer or by the non-Boltzmann nature of the discharge. Therefore, the estimated vibrational temperature here is the temperature corresponding to the minimum residual error for the three bands considered. As shown in figure 11, estimated vibrational temperatures of $3460 \pm 100 \mathrm{~K}$ at atmospheric pressure and $3980 \pm 100 \mathrm{~K}$ at $p=50 \mathrm{mbar}$ can be extracted. Obviously, the vibrational temperature is considerably higher than the rotational and, presumably, the translational temperatures. The reduction of the vibrational temperature with increasing operating pressure can be attributed to two factors. On the one hand, there is enhanced collisional relaxation of the vibrationally excited levels as the collisional frequency increases. On the other hand, less energy may be put into the vibrational excitation due to the increase in the rotational excitation energy, as discussed in section 3.3.1.

In order to illustrate the discharge behavior during the pulse duration, the time evolution of the spectral intensity between $330-420 \mathrm{~nm}$ over a time span of $80 \mathrm{~ns}$ is shown in figure 12. Each spectrum in this figure is integrated over a temporal window of $3 \mathrm{~ns}$, using a 300 grooves $/ \mathrm{mm}$ grating. As shown, there are two main peaks in the spectra, separated by $40 \mathrm{~ns}$. This behavior agrees very well with the power measurements from figure 7(b), as it also shows two principle power peaks with roughly the same time separation.

It is worth mentioning that no vibrational or rotational temperature differences were observed between the incident pulse at $t=10 \mathrm{~ns}$ and the reflected pulse at $50 \mathrm{~ns}$, as can be seen in figure 13. These spectra have been recorded in stagnant air at atmospheric pressure using the 1800 grooves/ $\mathrm{mm}$ grating centered at $355 \mathrm{~nm}$ to cover the (0-1) and (1-2) bands of the SPS of $\mathrm{N}_{2}$. About 30 percent reduction in emission intensity has been observed between the incident and reflected pulses.

3.3.3. Average gas temperature. To measure the average gas temperature downstream of the reactor, we have used a thermocouple type K (model Testo 0613-1912) with a $5 \mathrm{~mm}$ probe diameter and reaction time of 35 seconds to damp the noise. The probe junction is located $5 \mathrm{~mm}$ downstream from the central hole of the reactor. Figure 14 shows the air 

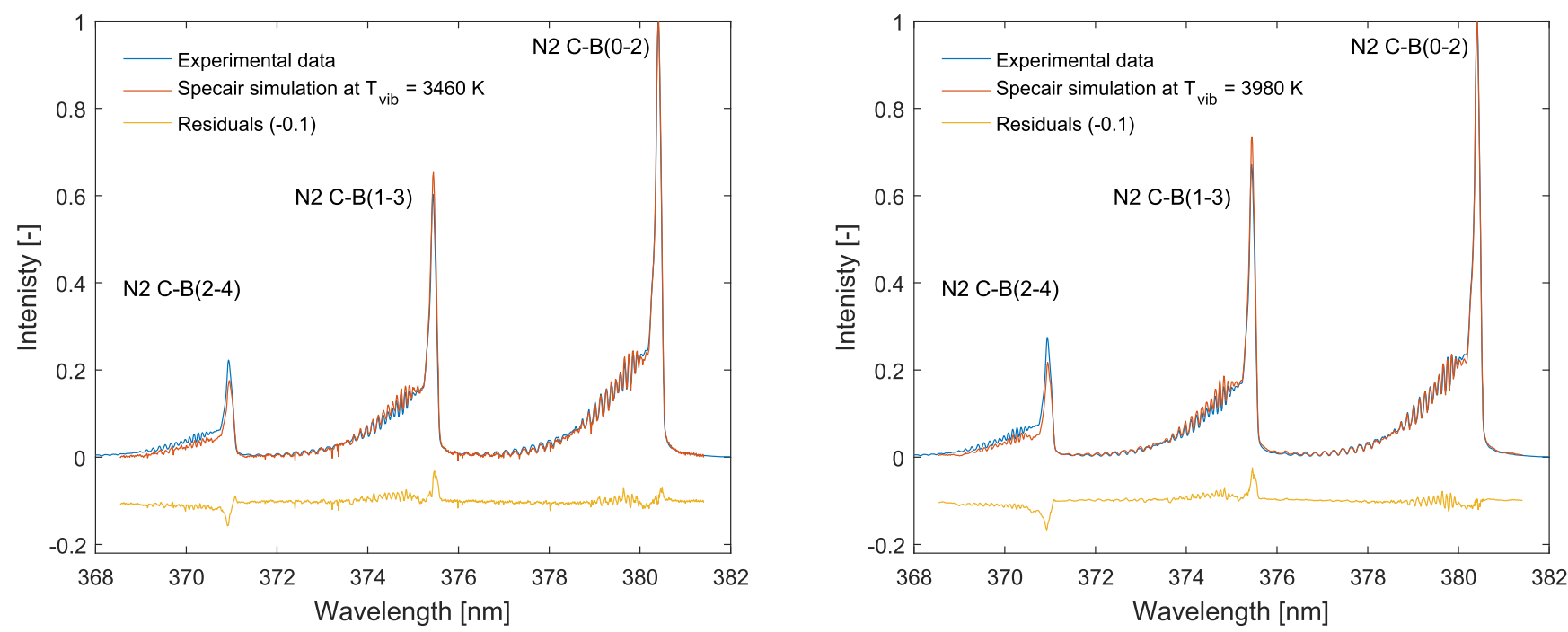

Figure 11. Experimental (blue line) and simulated (red line) normalized spectra of stagnant air at (left) $p=1$ bar, and (right) $p=50 \mathrm{mbar}$. Simulated spectra are from Specair with $T_{\mathrm{vib}}=3460 \mathrm{~K}$ and $T_{\mathrm{vib}}=3980 \mathrm{~K}$, respectively. Both are for $V=5 \mathrm{kV}$ and pulse repetition rate of $3 \mathrm{kHz}$. Signals are integrated over 100,000 pulses using a 1800 grooves $/ \mathrm{mm}$ grating.

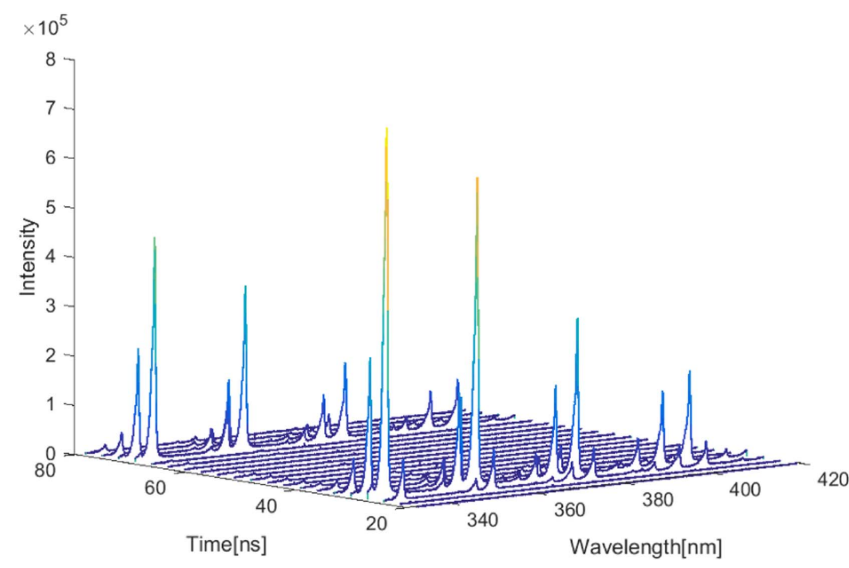

Figure 12. Temporal development of emission spectra of the plasma discharge at atmospheric pressure in air, with an integration window of $3 \mathrm{~ns}$ using a 300 grooves $/ \mathrm{mm}$ grating.

temperature as a function of pulse repetition frequency for an air flow rate of $3.41 \mathrm{~min}^{-1}$ at atmospheric pressure.

As shown in the figure, there is a linear relationship between the pulse repetition frequency and average gas temperature, with a slope of $6.5 \mathrm{~K} \mathrm{kHz}^{-1}$. From this relationship, we can extract an average value of the thermal power generated by the plasma discharge, $Q_{\text {plasma }}=\dot{m} \cdot c_{\mathrm{p}} \cdot \Delta T$, where $\dot{m}$ is the mass flow rate, $c_{\mathrm{p}}$ is the specific heat of air at constant pressure and $\Delta T$ is the difference between inflow and outflow temperatures. This gives an average value of $0.45 \mathrm{~W}$ for $5 \mathrm{kV}$ pulses at a repetition frequency of $1 \mathrm{kHz}$, or in other words, the energy spent on gas heating is $0.45 \mathrm{~mJ} /$ pulse for all channels combined.

This value is $85 \%$ of the pulse energy calculated from the voltage and current measurements at atmospheric pressure in section 3.1. The rest of the consumed power is spent on other degrees of freedom such as dissociation of the molecular gas components (such as the dissociation of the molecular oxygen

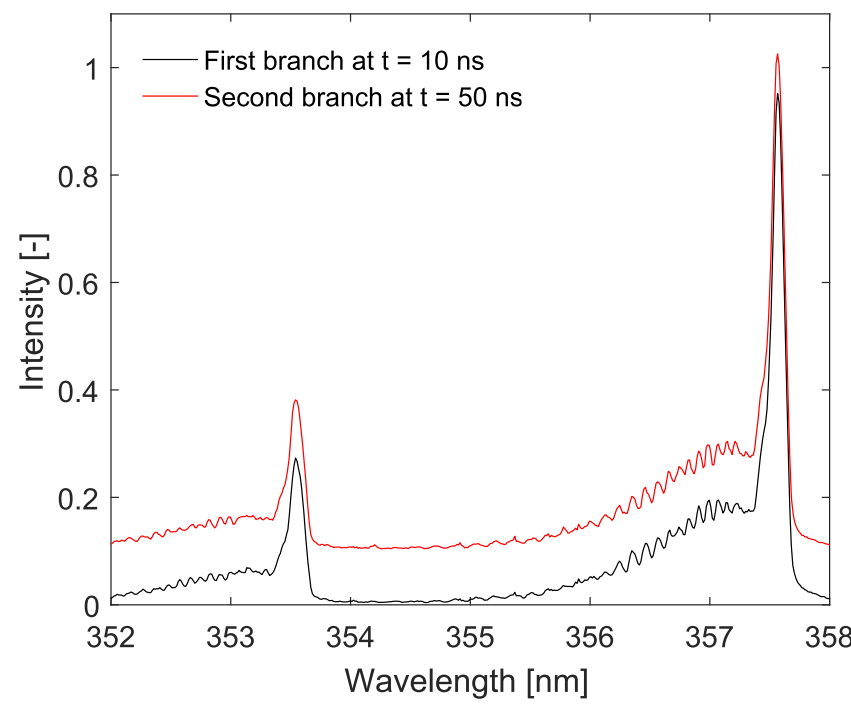

Figure 13. Comparison of the normalized spectra for the incident pulse (black line) and the reflected pulse (red line) of the pulse. Signals are integrated over 100,000 pulses for stagnant air at atmospheric pressure. Second peak line has been shifted upward by 0.1 for better visualization.

in the quenching reaction of $\mathrm{N}_{2}$ (B) [30]), heating of the dielectric material and conduction to the metal body. In the case of reduced pressure, it is expected that the fraction of the power, which is dissipated into gas heating will be lower, and more power will go to the chemical processes due to the higher electron energy and hence ionization efficiency.

\subsection{Effective reduced electric field strength $(E / N)_{\mathrm{eff}}$}

Determination of the actual reduced electric field $(E / N)$ by direct methods is relatively complicated for pulsed microdischarges because both the electric field $(E)$ and the concentration of neutral particles $(N)$ vary strongly in time and space. However, Paris et al [31] introduced an indirect 


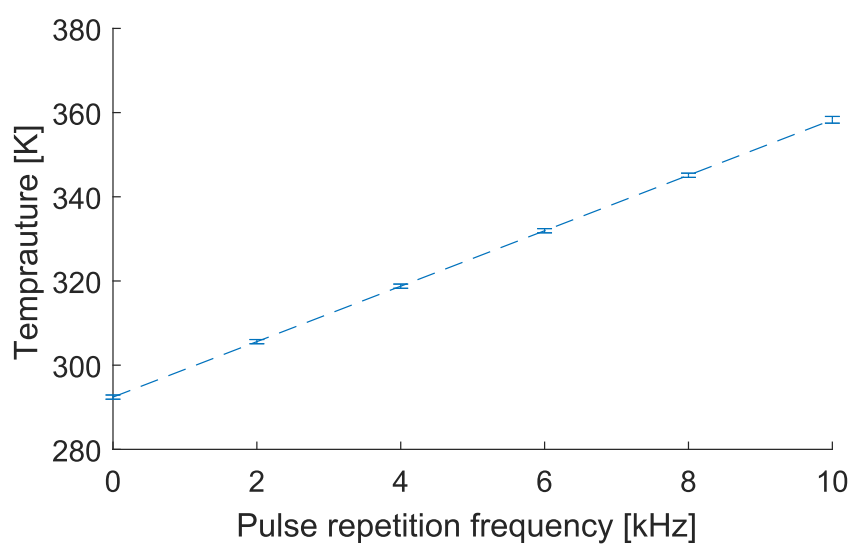

Figure 14. Measured gas temperature as a function of pulse repetition frequency for air with flow rate of $3.41 \mathrm{~min}^{-1}$ at atmospheric pressure and $V=5 \mathrm{kV}$. Dashed line indicates a linear fit and the error bars represent the standard deviation in the measurements.

method by using the intensity ratio of nitrogen spectral bands. For this, the (0-0) transition band of the first negative system of $\mathrm{N}_{2}^{+}$(FNS; $B^{2} \Sigma_{u}^{+} \rightarrow X^{2} \Sigma_{g}^{+}$) at $391.4 \mathrm{~nm}$ and the SPS (2-5) band at $394.3 \mathrm{~nm}$ are used. The following relation between the intensity ratio $R_{391 / 394}$ and the reduced electric field has been proposed for low-temperature plasmas in air at a pressure range of $0.3-100 \mathrm{kPa}$ with a maximum deviation of $12 \%$ :

$$
\mathrm{R}_{391 / 394}=46 \exp \left[-89\left(\frac{E}{N}\right)^{-0.5}\right] .
$$

The distribution of the $\mathrm{N}_{2}^{+}(B-X)(0-0)$ and $\mathrm{N}_{2}(C-\mathrm{B})$ (2-5) intensities over the channel width are presented in figure 15, at 300 and $50 \mathrm{mbar}$, for both the incident and reflected pulses at $5 \mathrm{kV}$ and $3 \mathrm{kHz}$ pulse repetition rate. The spectra of the $\mathrm{N}_{2}^{+}(B-X)(0-0)$ and $\mathrm{N}_{2}(C-\mathrm{B})(2-5)$ bands are accumulated for $10 \mathrm{~ns}$ after the onset of each pulse and integrated over 300,000 pulses using a 1800 grooves $/ \mathrm{mm}$ grating.

Three distinct regimes can be recognized from these figures when going from the center of a channel outwards: (1) inside the channel (A-B), (2) between the channel and the grounded electrode edge (B-C), and (3) the grounded electrode (C-D). At 300 mbar, and during the incident pulse, the minimum intensity of the nitrogen FNS is located at the center of the channel (point A). Going outwards, two peaks can be distinguished. The first peak is located inside the channel close to the wall (point B). This is caused by the integration over the line of sight of the ionization wave, which is traveling from the high-voltage electrode towards the channel exit. This peak is expanding by reducing the pressure until it reaches the center at 50 mbar when the central minimum disappears.

The second peak is located at the grounded electrode edge (point $\mathrm{C}$ ), which is most likely produced by a discharge, which is formed due to the field enhancement at the grounded electrode edge. The intensities of the two peaks have almost the same strength, even at different operating pressures. In contrast, during the reflected pulse, only one peak of $\mathrm{N}_{2}^{+}(B-X)(0-0)$ is formed, the one at the channel walls,
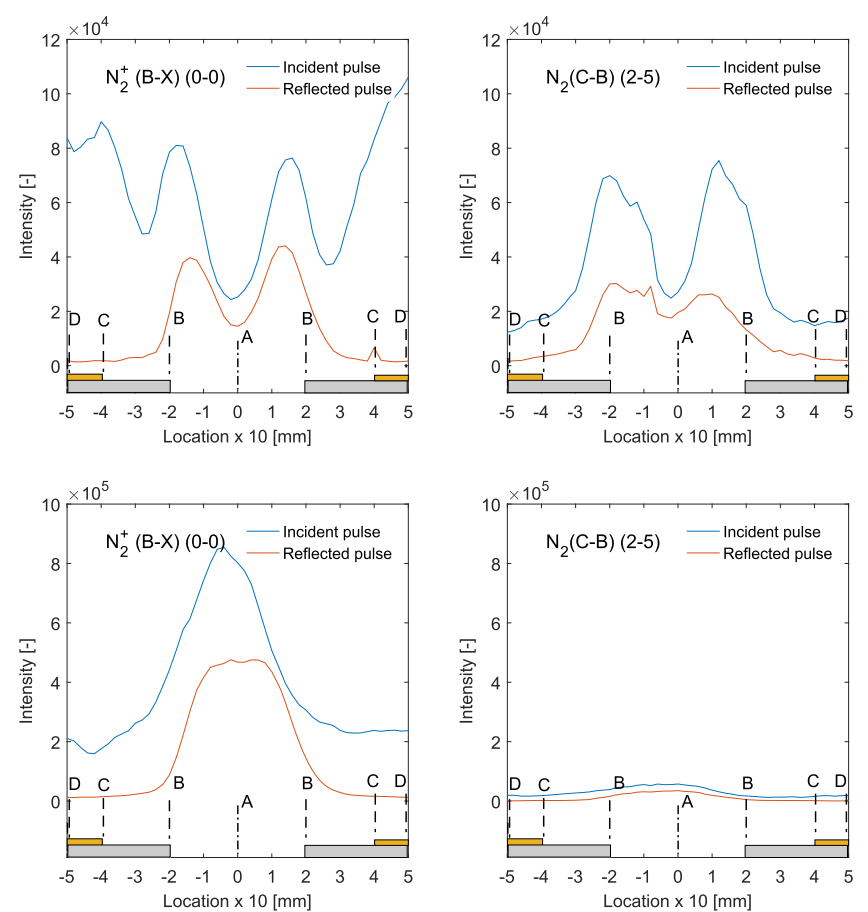

Figure 15. Emission intensity of (left) the $\mathrm{N}_{2}^{+}(B-X)(0-0)$ at $391.4 \mathrm{~nm}$ and (right) the $\mathrm{N}_{2}(C-\mathrm{B})(2-5)$ at $394.3 \mathrm{~nm}$ over one channel at pressures of 300 mbar (top) and 50 mbar (bottom). Signals are accumulated for $10 \mathrm{~ns}$ during the incident pulse (in blue) and reflected pulse (in red). All are for $V=5 \mathrm{kV}$ and $3 \mathrm{kHz}$ repetition rate in stagnant air. Signals are integrated for 300,000 pulses.

while the one at the grounded edge is absent. This could be explained by the following three effects. First, the high electron density left over from the incident pulse around the grounded electrode covers the sharp edge, which consequently reduces the local field enhancement as well as the energy and number of produced electrons. Second, it could be related to the drift direction of the electrons. Third, the low voltage of the reflected pulse compared to the incident pulse may prevent the $\mathrm{N}_{2}^{+}(B-X)(0-0)$ excitation.

The vibrationally excited nitrogen state $\mathrm{N}_{2}(C-B)(2-5)$ appears mostly near the wall of the channel during both the incident and reflected pulses, although with about half of the intensity during the reflected pulse. Furthermore, it seems like the discharge at the grounded electrode, near point $(\mathrm{C})$, consumes most of the energy on the ionization and less on vibrational excitation. This can explain the reduced amount of $\mathrm{N}_{2}(C-B)(2-5)$ compared to $\mathrm{N}_{2}^{+}(B-X)(0-0)$ at this location.

For pressures between one atmosphere and 100 mbar, the discharges have almost identical structures and only differ in the relative intensities between the excited states. However, at 50 mbar, the structure changes dramatically, as shown in the bottom graphs in figure 15. The major difference in the structure is that there is only one peak in the center of the channel for both the SPS of $\mathrm{N}_{2}$ and FNS of $\mathrm{N}_{2}^{+}$. This is due to the large main free path at 50 mbar, as discussed earlier in 


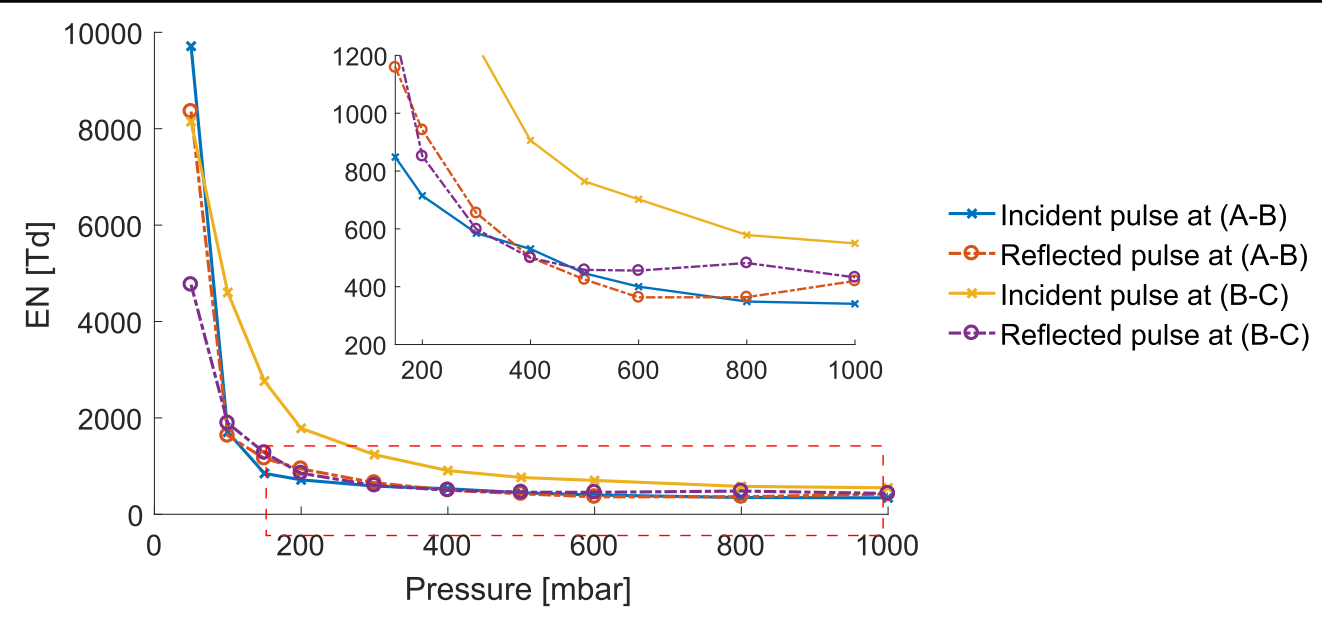

Figure 16. Effective reduced electric field $(E / N)_{\text {eff }}$ as a function of the operating pressure, calculated from the intensity ratio $R_{391 / 394}$ for (solid line) incident pulse, (dashed line) reflected pulse, $(\times)$ for B-C, and (o) for A-B. Inset shows a magnification for the range of 150 mbar1 bar. Plasma conditions are given in figure 15.

section 3.2. It is also clear that the FNS of $\mathrm{N}_{2}^{+}$is about one order of magnitude higher than the SPS of $\mathrm{N}_{2}$, which indicates a high reduced electric field strength.

Figure 16 shows the effective reduced electric field strength $(E / N)_{\text {eff }}$, which is determined by the intensity ratio method, presented in equation (3), for the incident and reflected pulses. A spatial averaging has been performed for the $\mathrm{N}_{2}^{+}(B-X)(0-0)$ and $\mathrm{N}_{2}(C-B)(2-5)$ over (A-B) and (B-D) positions for one channel. The signals are accumulated for $10 \mathrm{~ns}$ and integrated over the line of sight for 300,000 pulses.

The general trend, for all lines in the figure, is that the effective reduced electric field strength increases gradually with decreasing operating pressure. This trend is expected, as the neutral number density of the gas decreases with pressure, while the applied voltage (and therefore the path-integrated electric field) remains constant. The figure shows a value of $550 \pm 100 \mathrm{Td}$ and $340 \pm 50 \mathrm{Td}$ for $(E / N)_{\text {eff }}$ for the incident pulse inside the channel (A-B) and outside the channel (B-D), respectively. By reducing the pressure to $100 \mathrm{mbar},(E / N)_{\text {eff }}$ increases gradually to $4600 \pm 700 \mathrm{Td}$ and $1900 \pm 300 \mathrm{Td}$. Also, at this pressure range, $(E / N)_{\text {eff }}$ for the reflected pulse inside the channel is slightly higher than for the incident pulse. This can be explained by the substantial increase in $(E / N)_{\text {eff }}$ of the incident pulse outside the channel. By reducing the pressure down to $50 \mathrm{mbar},(E / N)_{\text {eff }}$ inside the channel increases to about $10000 \pm 1500 \mathrm{Td}$ for the incident pulse with a relatively lower value outside the channel.

\subsection{Effect of flow rate on discharge emission}

To study the effect of the flow velocity inside the holes on the discharge intensity in the DBD microplasma reactor, a set of experiments has been done at different air flow velocities at atmospheric pressure with $5 \mathrm{kV}$ at $3 \mathrm{kHz}$ repetition rate. This study has been carried out for mean air flow velocities ranging from $0-7 \mathrm{~m} \mathrm{~s}^{-1}$ inside the channels, corresponding to average flow velocities from $0-1 \mathrm{~m} \mathrm{~s}^{-1}$ above the reactor.
The residence time of a volume of gas passing through the discharge region in a channel (length of $0.36 \mathrm{~mm}$ for single-layer operation) ranges from $50-250 \mu$ s for air velocities between $7-1.4 \mathrm{~m} \mathrm{~s}^{-1}$. Obviously, the minimum residence time is much longer than the characteristic time scale of the plasma discharge, which is on a tens of nanosecond time scale. However, these residence times are slightly shorter than the inter-pulse delay at a pulse repetition frequency of $3 \mathrm{kHz}$. This can explain the reduction in emission intensity at higher flow velocities, as shown in figure 17. The figure shows a roughly $25 \%$ reduction in emission intensity when increasing the flow from 0 to $1.4 \mathrm{~m} \mathrm{~s}^{-1}$ and a smaller, more gradual, reduction when increasing the flow further up to $7 \mathrm{~m} \mathrm{~s}^{-1}$. In stagnant air, all high-voltage pulses ionize the same volume of gas in the discharge region. This increases the ionization level before the new breakdown occurs, which leads to higher emission intensity [32,33], while at higher velocities most of the gap is filled with fresh gas and less pre-ionization will exist.

\subsection{Reactor lifetime assessment}

One of the serious problems of microplasma devices is their often short lifetime due to surface and internal damage. This is due to the frequent loss of plasma confinement due to vaporization and ablation, which cause significant surface erosion and structural failure [34, 35]. Reactor lifetime is very sensitive to many parameters, e.g. geometry, material of the dielectric and power source.

Figure 18 shows the dependence of the reactor lifetime on the pulse repetition frequency at atmospheric pressure and 50 mbar. At a frequency of $2 \mathrm{kHz}$ and atmospheric pressure, the reactor lifetime was about $24 \mathrm{~min}$ in continuous operation mode. While at $50 \mathrm{mbar}$, it was about $100 \mathrm{~min}$. This shows an enhancement in the reactor lifetime of about four times by lowering the operating pressure by a factor of 20 . This enhancement increases to a factor of about ten at a pulse repetition rate of $10 \mathrm{kHz}$, even though the lifetime itself decreases in both cases. 


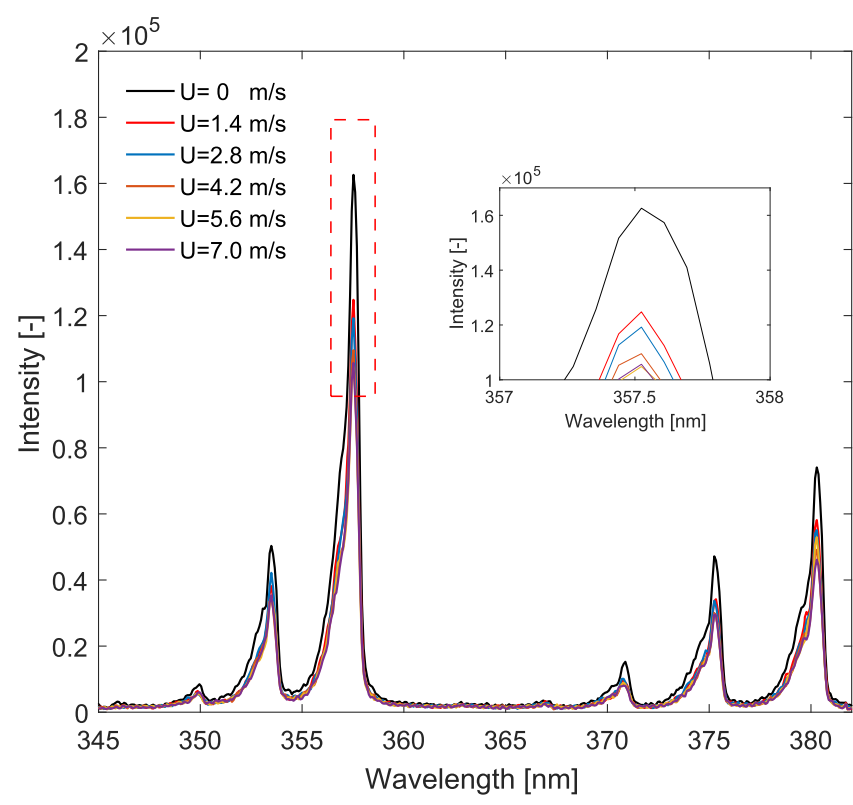

Figure 17. Non-normalized emission spectra in air at atmospheric pressure for different flow velocities for $5 \mathrm{kV}$ and $3 \mathrm{kHz}$ repetition rate. Inset shows a magnification of the largest peaks (dashed).

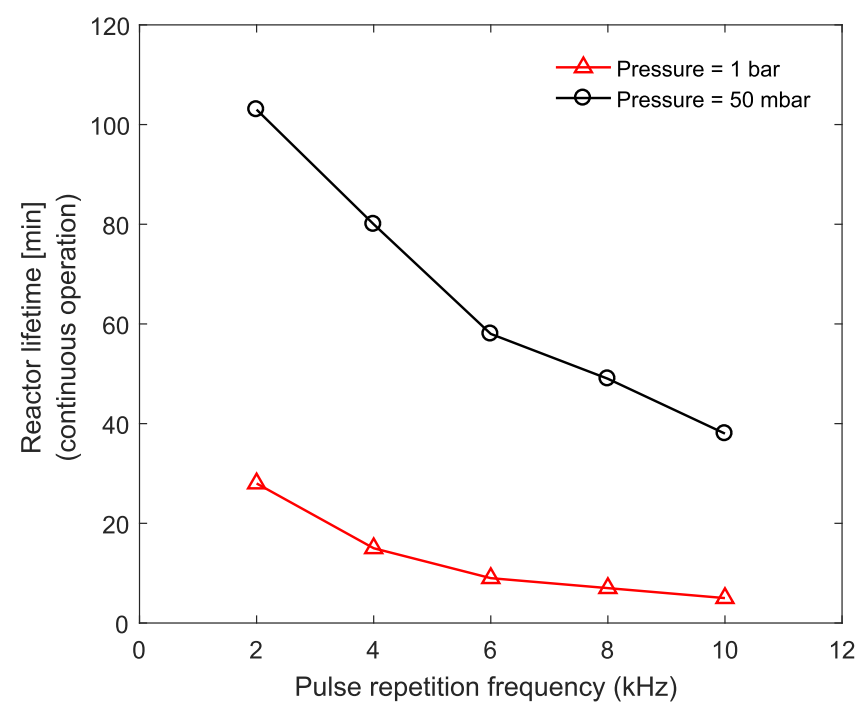

Figure 18. Reactor lifetime in continuous operation mode as a function of pulse repetition frequency at atmospheric pressure and 50 mbar.

Two factors may contribute to the observed enhancement. First, at low pressure, the ion impact frequency is low, which slows down the dielectric wear-down process. Second, at low pressure the gas temperature is lower, as was shown in section 3.3.1. Both reasons make the reactor more prone to failure at atmospheric pressure due to thermal stress on the internal structure of the dielectric material. Other factors that may affect the lifetime of the reactor and need more investigation are voltage amplitude and duration, geometry, reactor material and operating gas.

\section{Summary and conclusion}

In this work, a new DBD microplasma flow reactor driven by nanosecond high-voltage pulses has been presented. With this design we were able to sustain a non-equilibrium plasma discharge in a pattern of $400 \mu \mathrm{m}$ diameter channels at pressures up to 1 bar. The DBD microplasma flow reactor is a promising device for a wide range of applications that require an efficient interaction between a non-thermal plasma discharge and a gas flow. Some of these applications are pollutant control, $\mathrm{CO}_{2}$ to $\mathrm{CO}$ conversion, plasma-assisted combustion and plasma medicine.

Time-resolved electrical and optical measurements have been conducted to characterize the main features of the plasma discharge in the DBD microplasma reactor. Pulse energies per channel of about 1.46 and $1.3 \mu \mathrm{J}$ have been calculated from the current and voltage time evolution at atmospheric pressure and 50 mbar, respectively. The discharge at low pressure is characterized by high vibrational temperatures (roughly $4000 \mathrm{~K}$ ) and high effective reduced electric field strengths (10000 Td) compared to atmospheric pressure $(3460 \mathrm{~K}$ and $550 \mathrm{Td})$, which indicates a higher electron energy at lower pressure. Based on luminosity images, all gas flowing through the reactor can be assumed to interact with the plasma at 50 mbar. At higher pressures, the emission concentrates at the channel walls and not all gas is in direct contact with the plasma. The distribution of the plasmagenerated radicals, however, is unknown. The luminous part of the plasma is strongly non-thermal, with vibrational temperatures of about $4000 \mathrm{~K}$, but the gas temperature remains only a few degrees above ambient.

In addition, we have noticed that the discharge emission intensity decreases slightly with increasing air flow velocity through the channels at atmospheric pressure due to the lower pre-ionization level at higher velocities. Finally, a reactor lifetime study showed around 100 min lifetime at low pressure. Nevertheless, more effort is needed to increase the lifetime of the reactor in order to transfer it to real applications.

\section{Acknowledgments}

A Elkholy is supported by a $\mathrm{PhD}$ grant from the Ministry of Higher Education, Government of Egypt.

\section{ORCID iDs}

A Elkholy (i) https://orcid.org/0000-0001-6215-6367

S Nijdam (1) https://orcid.org/0000-0002-1310-6942

\section{References}

[1] Shimizu K, Umeda A and Blajan M 2011 Surface treatment of polymer film by atmospheric pulsed microplasma: study on 
gas humidity effect for improving the hydrophilic property Japan. J. Appl. Phys. 50 08KA03

[2] Benedikt J, Raballand V, Yanguas-Gil A, Focke K and von Keudell A 2007 Thin film deposition by means of atmospheric pressure microplasma jet Plasma Phys. Control. Fusion 49 B419

[3] Shimizu K, Ishii T and Blajan M 2010 Emission spectroscopy of pulsed power microplasma for atmospheric pollution control IEEE Trans. Ind. Appl. 46 1125-31

[4] Shimizu K, Sugiyama T and Samaratunge M N 2008 Study of air pollution control by using micro plasma filter IEEE Trans. Ind. Appl. 44 506-11

[5] Lee H J, Shon C H, Kim Y S, Kim S, Kim G C and Kong M G 2009 Degradation of adhesion molecules of G361 melanoma cells by a non-thermal atmospheric pressure microplasma New J. Phys. 11115026

[6] Hensel K, Machala Z and Tardiveau P 2009 Capillary microplasmas for ozone generation Eur. Phys. J. Appl. Phys. 4722813

[7] Kolb J F, Mohamed A-A H, Price R O, Swanson R J, Bowman A, Chiavarini R, Stacey M and Schoenbach K 2008 Cold atmospheric pressure air plasma jet for medical applications Appl. Phys. Lett. 92241501

[8] Schutze A, Jeong J Y, Babayan S E, Park J, Selwyn G S and Hicks R F 1998 The atmospheric-pressure plasma jet: a review and comparison to other plasma sources IEEE Trans. Plasma Sci. 26 1685-94

[9] Chen H L, Lee H M, Chen S H and Chang M B 2008 Review of packed-bed plasma reactor for ozone generation and air pollution control Ind. Eng. Chem. Res. 47 2122-30

[10] Yu Q, Kong M, Liu T, Fei J and Zheng X 2012 Characteristics of the decomposition of $\mathrm{CO}_{2}$ in a dielectric packed-bed plasma reactor Plasma Chem. Plasma Process. 32 153-63

[11] Grabowski L, Van Veldhuizen E, Pemen A and Rutgers W 2007 Breakdown of methylene blue and methyl orange by pulsed corona discharge Plasma Sources Sci. Technol. 16226

[12] Ono R and Oda T 2003 Dynamics of ozone and OH radicals generated by pulsed corona discharge in humid-air flow reactor measured by laser spectroscopy J. Appl. Phys. 93 5876-82

[13] Winands G, Yan K, Pemen A, Nair S, Liu Z and Van Heesch E 2006 An industrial streamer corona plasma system for gas cleaning IEEE Trans. Plasma Sci. 34 2426-33

[14] Akitsu T, Ohkawa H, Tsuji M, Kimura H and Kogoma M 2005 Plasma sterilization using glow discharge at atmospheric pressure Surf. Coat. Technol. 193 29-34

[15] Gomes M, Sismanoglu B and Amorim J 2009 Characterization of microhollow cathode discharges Braz. J. Phys. 39 25-30

[16] Schoenbach K H, El-Habachi A, Shi W and Ciocca M 1997 High-pressure hollow cathode discharges Plasma Sources Sci. Technol. 6468

[17] Schoenbach K H, Verhappen R, Tessnow T, Peterkin F and Byszewski W 1996 Microhollow cathode discharges Appl. Phys. Lett. 68 13-5

[18] Foest R, Schmidt M and Becker K 2006 Microplasmas, an emerging field of low-temperature plasma science and technology Int. J. Mass Spectrom. 248 87-102
[19] Becker K, Schoenbach K and Eden J 2006 Microplasmas and applications J. Phys. D: Appl. Phys. 39 R55

[20] Papadakis A P, Rossides S and Metaxas A C 2011 Microplasmas: a review Open Appl. Phys. J. 4 45-63

[21] Sakai O, Kishimoto Y and Tachibana K 2005 Integrated coaxial-hollow micro dielectric-barrier-discharges for a large-area plasma source operating at around atmospheric pressure J. Phys. D: Appl. Phys. 38431

[22] Zhang S, Wang W-C, Jiang P-C, Yang D-Z, Jia L and Wang S 2013 Comparison of atmospheric air plasmas excited by high-voltage nanosecond pulsed discharge and sinusoidal alternating current discharge J. Appl. Phys. 114163301

[23] Pai D Z, Stancu G D, Lacoste D A and Laux C O 2009 Nanosecond repetitively pulsed discharges in air at atmospheric pressure- the glow regime Plasma Sources Sci. Technol. 18045030

[24] Sansonetti C J, Salit M L and Reader J 1996 Wavelengths of spectral lines in mercury pencil lamps Appl. Opt. 35 74-7

[25] Sismanoglu B, Grigorov K, Caetano R, Rezende M and Hoyer Y 2010 Spectroscopic measurements and electrical diagnostics of microhollow cathode discharges in argon flow at atmospheric pressure Eur. Phys. J. D 60 505-16

[26] Bruggeman P, Sadeghi N, Schram D and Linss V 2014 Gas temperature determination from rotational lines in nonequilibrium plasmas: a review Plasma Sources Sci. Technol. 23023001

[27] Roupassov D, Nikipelov A, Nudnova M and Starikovskii A Y 2009 Flow separation control by plasma actuator with nanosecond pulsed-periodic discharge AIAA J. 47168

[28] Laux C, Spence T G, Kruger C H and Zare R N 2003 Optical diagnostics of atmospheric pressure air plasmas Plasma Sources Sci. Technol. 12125

[29] Fridman A 2008 Plasma Chemistry (Cambridge: Cambridge University Press)

[30] Rusterholtz D, Lacoste D, Stancu G, Pai D and Laux C 2013 Ultrafast heating and oxygen dissociation in atmospheric pressure air by nanosecond repetitively pulsed discharges J. Phys. D: Appl. Phys. 46464010

[31] Paris P, Aints M, Valk F, Plank T, Haljaste A, Kozlov K V and Wagner H-E 2005 Intensity ratio of spectral bands of nitrogen as a measure of electric field strength in plasmas J. Phys. D: Appl. Phys. 383894

[32] Höft H, Becker M and Kettlitz M 2016 Impact of gas flow rate on breakdown of filamentary dielectric barrier discharges Phys. Plasmas 23033504

[33] Nijdam S, van de Wetering F M J H, Blanc R, van Veldhuizen E M and Ebert U 2010 Probing photoionization: experiments on positive streamers in pure gases and mixtures J. Phys. D: Appl. Phys. 43145204

[34] Felix V, Lefaucheux P, Aubry O, Golda J, von der Gathen V S, Overzet L J and Dussart R 2016 Origin of microplasma instabilities during de operation of silicon based microhollow cathode devices Plasma Sources Sci. Technol. 25025021

[35] Hassanein A and Konkashbaev I 1998 Performance and lifetime assessment of reactor wall and nearby components during plasma instabilities $J$. Nucl. Mater. 258 645-52 